\title{
Theory of Quadratic Optical Frequency Combs
}

\author{
Tobias Hansson *, ${ }^{*}$ François Leo ${ }^{+}$, Miro Erkintalo ${ }^{+}$, Stephane Coen $^{+}$, Iolanda Ricciardi ${ }^{\S}$, \\ Maurizio De Rosa ${ }^{\S}$, and Stefan Wabnitz ${ }^{\circ}$, Member, IEEE \\ * Department of Applied Physics, Chalmers University of Technology, SE-41296 Göteborg, Sweden \\ Dipartimento di Ingegneria dell'Informazione, Università di Brescia, via Branze 38, 25123 Brescia, Italy \\ + The Dodd-Walls Centre, Department of Physics, The University of Auckland, Auckland 1142, New Zealand \\ $\S$ CNR-INO, Istituto Nazionale di Ottica, Via Campi Flegrei 34, 80078 Pozzuoli (NA), Italy \\ Tel: (39030) 3715846,Fax: (39030)380014, e-mail: stefan.wabnitz@unibs.it
}

\begin{abstract}
We present theoretical studies of optical frequency comb generation in dispersive quadratically nonlinear resonators. We introduce a mean field equation approach to model cavity enhanced second harmonic generation and find excellent agreement with recent experimental frequency comb observations. We also develop a more general approach based on a single envelope equation for both quadratic and Kerr nonlinear cavities.
\end{abstract}

Keywords: nonlinear optics, parametric processes, parametric oscillators, optical resonators.

\section{INTRODUCTION}

The generation of an optical frequency comb (OFC) by means of a nonlinear microresonator shows promise for enabling chip scale devices that can complement current comb sources based on bulk mode-locked lasers [1]. Microresonator-based OFC sources can provide a direct link between optical and microwave frequencies and have a host of potential applications to metrology, including ultra-precise optical clocks, biomedical and environmental spectroscopy, microwave photonics and multi-user coherent optical communications.

A substantial boost to the advancement of the theory and numerical modelling of Kerr microresonator OFCs has been provided by the realization that one can model the dynamics of the system using a time-domain description. This approach is based on the mean-field driven and damped nonlinear Schrödinger equation [2], which was earlier derived for coherently pumped passive fiber cavities [3].

Most research to date has focused on coherently pumped cubic Kerr nonlinear microresonators, where the physical principle of OFC generation is cascade four-wave mixing activated by modulation instability. However, modulation instabilities and pulse trains can also be obtained from quadratic nonlinear cavities [4], that could allow frequency comb generation in wavelength ranges where pump sources are unavailable, in particular in the mid-infrared. At the same time, quadratic comb sources can also lead to a substantial decrease in the required pump power level and allow for chip scale integration.

In fact, recent experiments have demonstrated the possibility of achieving OFC generation through the singly resonant cavity second harmonic process [5,6]. In this work, we review our development of the theory of OFC generation in quadratic cavities and microresonators, based on time-domain nonlinear evolution equations with different levels of complexity [7-10]. To validate our theory, we show that an excellent agreement between the theory and the experimental results is achieved.

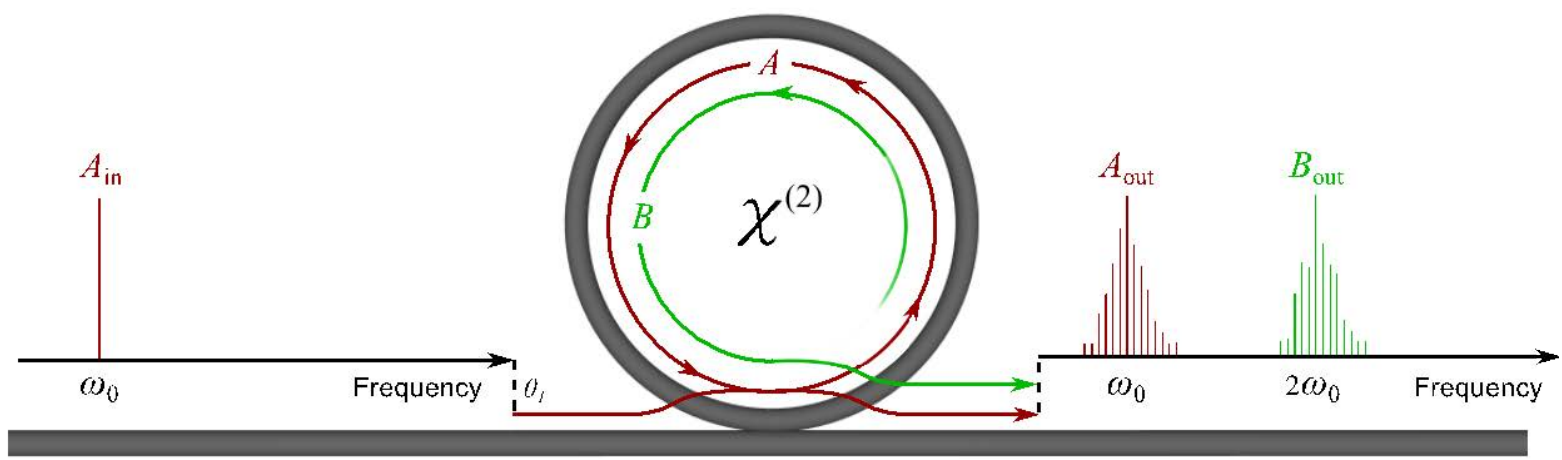

Figure 1. Schematic of OFC generation via SHG in a singly resonant quadratically nonlinear cavity.

\section{COUPLED EQUATIONS APPROACH}

In Fig. 1 we illustrate the basic architecture of OFC generation by means of a quadratic nonlinear resonator, pumped by a CW beam at the fundamental frequency (FF) $\omega_{0}$. The pump is coupled into the cavity where it generates its second harmonic $(\mathrm{SH}) 2 \omega_{0}$ : we suppose that a phase-matching mechanism is present such as periodic poling of the quadratic nonlinear material along the length of the resonator. In the scheme of Fig. 1, we consider a singly resonant cavity, where the FF re-circulates in the resonator, while the generated SH field leaves 
the resonator at each circulation. Above a certain threshold power, propagation of the FF and the $\mathrm{SH}$ in the cavity is modulationally unstable, which leads to the generation of a series of comb lines around both $\omega_{0}$ and $2 \omega_{0}$ through optical parametric oscillation and different cascaded quadratic processes.

OFC generation around both the FF and the SH can be described in general by means of two coupled maps that include separate propagation equations for frequency components around $\omega_{0}$ and $2 \omega_{0}$, along with the respective cavity boundary conditions $[7,11]$. Similar to the approach used for the modelling of Kerr combs, one may average the propagation over the length of the cavity, whenever the fields change relatively little over a single circulation inside the cavity, which leads one to obtain path-averaged (or mean-field) coupled equations that include the distributed presence of the boundary conditions.

Quite remarkably, under the physically relevant conditions of a large temporal walk-off with the FF and SH fields, the intra-cavity evolution of the SH is found to be effectively locked to the fundamental, whose temporal dynamics can be described by a single evolution equation with an effective non-instantaneous cubic nonlinearity

$$
\tau_{\mathrm{R}} \frac{\partial A(t, \tau)}{\partial t}=\left[-\alpha-i \delta-i L \frac{k^{\prime \prime}}{2} \frac{\partial^{2}}{\partial \tau^{2}}\right] A-\rho A^{*}\left[A^{2}(t, \tau) \otimes \mathrm{I}(\tau)\right]+\sqrt{\theta} A_{i n}
$$

Here $\tau_{\mathrm{R}}$ is the cavity circulation time, $A$ is the slowly varying envelope of the field centred at $\omega_{0}$, while $t$ and $\tau$ represent the slow time (proportional to the number of circulations), and the fast (or ordinary) time, respectively. Moreover, $\alpha$ is the distributed cavity loss and $\delta$ is the detuning of the cavity of length $L, k^{\prime \prime}$ is the group-velocity dispersion coefficient at the FF, $\rho$ is a nonlinear coupling coefficient, $\theta$ is the power transmission coefficient of the input coupler and $A_{\text {in }}$ is the driving input pump field. The function $I(\tau)$ is a nonlinear response function that takes different forms depending on whether the cavity is singly or doubly resonant, and the symbol $\otimes$ denotes convolution.

In Fig. 2 we compare the predictions of OFC generation via singly resonant phase-matched SHG as obtained by Eq. (1) (left column), with the corresponding experimental results (right column) for a bulk cavity containing a periodically poled lithium niobate crystal. In Figs. 2(a)-(a1) we show by red diamonds (squares) the theoretical (experimental) power in all the comb lines (signal/idler) around the FF: in both cases, the comb centred at $\omega_{0}$ is generated at a threshold input pump power of about $100 \mathrm{~mW}$. Figures 2(a)-(a1) also show by blue dots the $\mathrm{SH}$ power, revealing its clamping to a fixed value above the threshold pump power. Figures 2 (b,c,d) and (b1,c1,d1) show that excellent agreement is obtained between theoretical and experimental comb spectra around the FF, as either the input pump power or the cavity detuning is varied. In Fig. 2(e,f,g) we also illustrate the corresponding numerically computed combs that are generated around the $\mathrm{SH}$ for the same parameters.
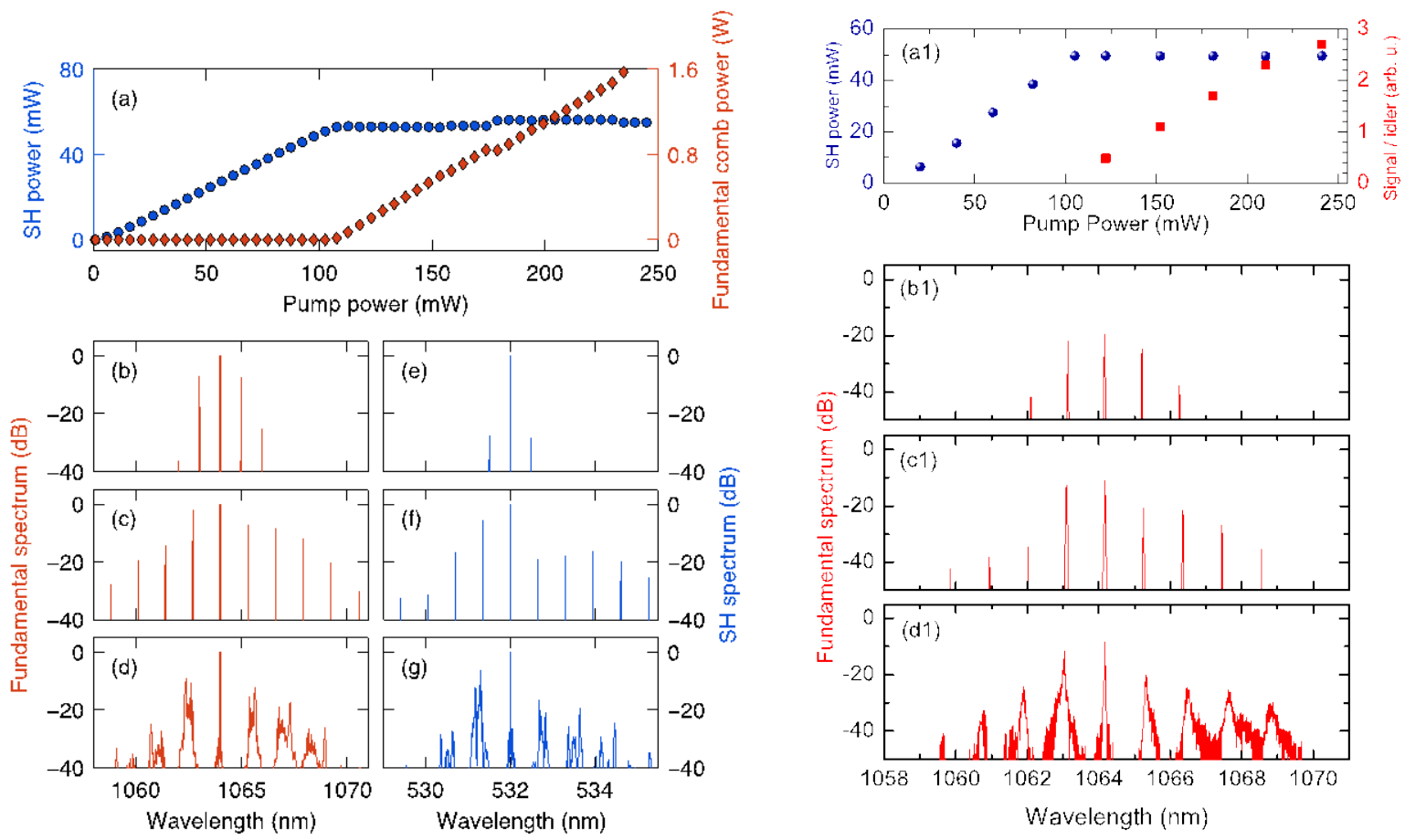

Figure 2. Comparison of numerically simulated (left column) and experimental (right column) OFC generation in singly resonant, phase-matched cavity $S H G$. 
Besides enabling efficient numerical simulations of the experiments, the mean-field model of Eq. (1) permits one to carry out a straightforward analytical study of the gain spectrum of modulation instabilities of the $\mathrm{CW}$ solutions of the quadratic cavity. Our analysis reveals that OFC generation occurs due to walk-off induced modulational instability in the regime of large group velocity mismatch between the FF and the $\mathrm{SH}$, a situation that has not been investigated in earlier studies of either spatially diffractive or temporally dispersive SHG cavities.

Similar to the case of Kerr combs, the time domain approach of Eq. (1) is largely equivalent to a frequency domain description involving a set of coupled cavity modes. Although the specific advantage of using either a time-domain or a frequency domain approach will depend on the particular application, the use of Eq. (1) generally simplifies both numerical and analytical treatments by allowing simultaneous and efficient modelling of large numbers of comb lines around both the fundamental and second harmonic.

\section{SINGLE ENVELOPE EQUATION APPROACH}

The previously described approach for modelling the temporal dynamics of quadratic OFC generation in terms of separate equations for the mean-field envelopes centred on different carrier frequencies (of the FF, the SH, or OPO) inherently assumes that there is no spectral overlap between the different combs. Whenever this condition is not satisfied, or when multiple wave mixing processes are simultaneously present, a single-envelope equation (SEE) approach should be used. In the frame of this approach, the main assumption is that of unidirectional wave propagation, however no slowly varying (with respect to the optical cycle) envelope approximation is made, so that one may describe nonlinear propagation of waves with a spectral extension of multiple octaves. Moreover, the presence of competing (e.g., cubic) nonlinearities, including nonlinear loss mechanisms, can be easily included in the SEE approach.
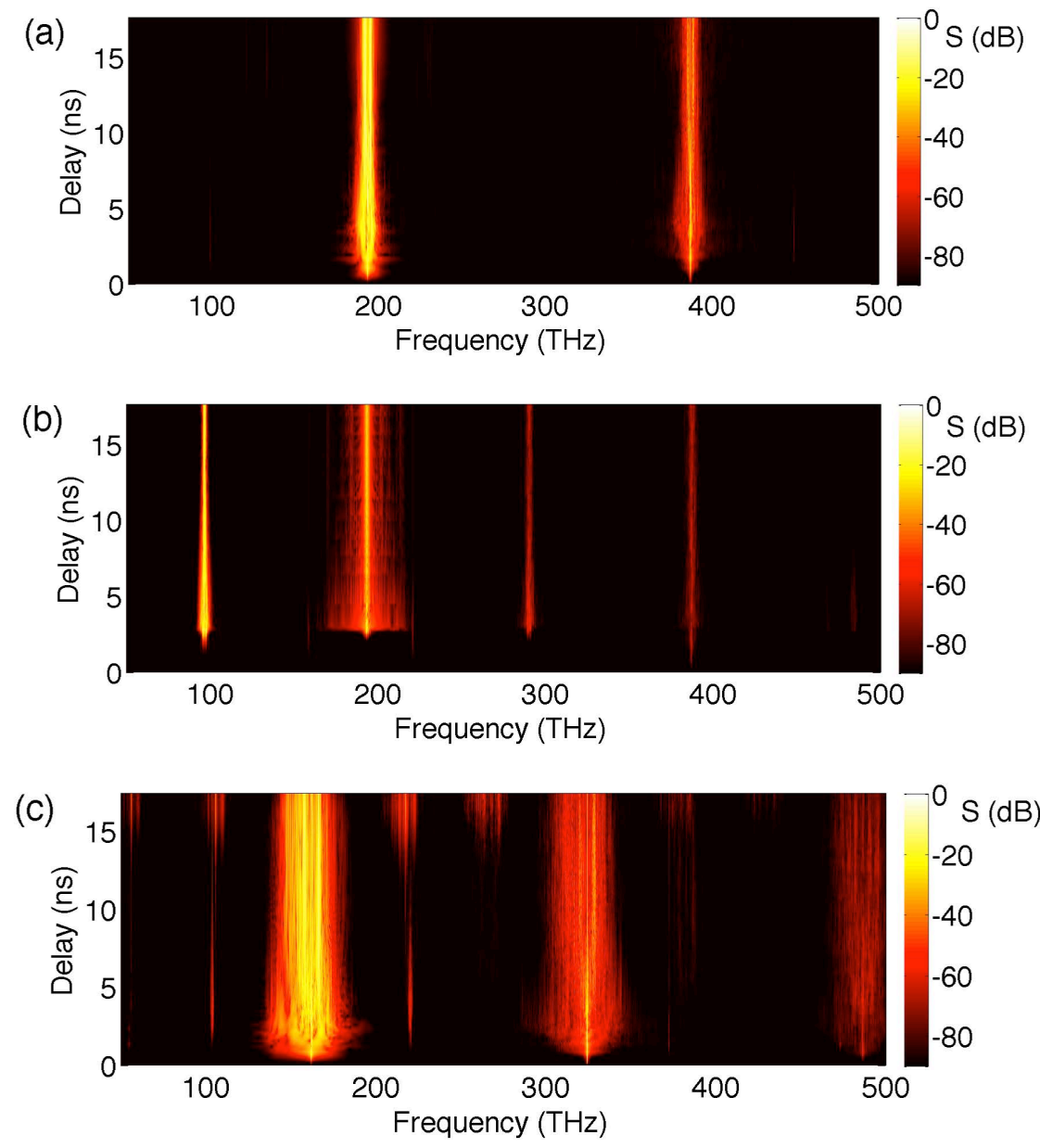

Figure 3. Spectral evolution of intra-cavity power showing OFC generation at the FF and: (a) the SH frequency;

(b) the degenerate OPO frequency; (c) the SH and the non-degenerate OPO frequencies.

In Fig. 3 we illustrate the spectral evolution (as a function of slow time $t$ ) of OFC generation in radially poled lithium niobate microresonator, when the crystal poling period is varied to phase-match different wave mixing processes. Figures 3(a) and 3(c) shows conditions where the cavity is phase-matched for SHG, while Fig. 3(b) considers phase-matching for OPO. The FF comb is centred at the pump frequency of $192 \mathrm{THz}$ and has a pump 
power equal to $20 \mathrm{~mW}$ in Figs. 3(a) and 3(b), whereas in Fig. 3(c) the pump frequency is moved to $162 \mathrm{THz}$ with a pump power of $100 \mathrm{~mW}$. As can be seen, in Fig. 3(a) two stable and well-separated combs are generated around the FF and the SH frequencies. Whereas in Fig. 3(b) the poling period leads to quasi-phase-matching (QPM) of the FF and the degenerate OPO frequency $\omega_{0} / 2$ : in addition to the main combs, weaker narrowband combs are also generated at the SH and the $3 \omega_{0} / 2$ frequencies. Finally, in the case of Fig. $3(\mathrm{c})$, the same poling period leads to QPM of SHG, and optical parametric generation of signal and idler waves at $41 \mathrm{THz}$ and $106 \mathrm{THz}$, respectively. Interestingly, Fig. 3(c) also shows the generation of weak secondary combs resulting from sum-frequency or difference-frequency generation processes. For example, the sum frequency process of the FF and the idler generates a comb at $218 \mathrm{THz}$, and the difference frequency process of the $\mathrm{SH}$ and the idler generates a secondary comb at $268 \mathrm{THz}$.

\section{CONCLUSIONS}

In summary, we have derived nonlinear wave equations for describing the temporal dynamics of optical frequency comb generation in quadratic nonlinear cavities. These models exhibit different levels of complexity and range from a single envelope equation approach that includes in the same envelope the presence of all possible mixing processes, to mean-field equations that describe individual combs generated around each of the main interacting waves. Remarkably, a single equation involving a non-instantaneous cubic nonlinear response function can be derived to describe the comb generated at the fundamental frequency in the latter case. The validity of the models has been validated by comparisons with experimental results.

\section{ACKNOWLEDGEMENTS}

We acknowledge support from the Italian Ministry of University and Research (MIUR) (2012BFNWZ2, and Progetto Premiale QUANTOM Quantum Opto-Mechanics); Swedish Research Council (SRC) (2013-7508); Rutherford Discovery Fellowships of the Royal Society of New Zealand; Marsden Fund of The Royal Society of New Zealand.

\section{REFERENCES}

[1] P. Del'Haye, A. Schliesser, O. Arcizet, T.Wilken, R. Holzwarth, and T. J. Kippenberg: Optical frequency comb generation from a monolithic microresonator, Nature (London), vol. 450, 1214, 2007.

[2] S. Coen, H. G. Randle, T. Sylvestre, and M. Erkintalo: Modeling of octave-spanning Kerr frequency combs using a generalized mean-field Lugiato-Lefever model, Opt. Lett., vol. 38, 37, 2013.

[3] M. Haelterman, S. Trillo, and S. Wabnitz: Dissipative modulation instability in a nonlinear dispersive ring cavity, Opt. Commun., vol. 91, 401 (1992).

[4] S. Trillo, M. Haelterman: Pulse-train generation through modulational instability in intracavity secondharmonic generation, Opt. Lett., vol. 21, 1114, 1996.

[5] I. Ricciardi, S. Mosca, M. Parisi, P. Maddaloni, L. Santamaria, P. De Natale, and M. De Rosa: Frequency comb generation in quadratic nonlinear media, Phys. Rev. A, vol. 91, 063839, 2015.

[6] S. Mosca, I. Ricciardi, M. Parisi, P. Maddaloni, L. Santamaria, P. De Natale, and M. De Rosa: Direct generation of optical frequency combs in $\chi^{(2)}$ nonlinear cavities, Nanophotonics, in press (2016) arXiv:1510.08074 [physics.optics].

[7] F. Leo, T. Hansson, I. Ricciardi, M. De Rosa, S. Coen, S. Wabnitz, and M. Erkintalo: Walk-off-induced modulation instability, temporal pattern formation, and frequency comb generation in cavity-enhanced second- harmonic generation, Phys. Rev. Lett., vol. 116, 033901, 2016.

[8] F. Leo, T. Hansson, I. Ricciardi, M. De Rosa, S. Coen, S. Wabnitz, and M. Erkintalo: Frequency comb formation in doubly resonant second-harmonic generation, Phys. Rev. A, in press, arXiv:1602.03517 [physics.optics], 2016

[9] T. Hansson, F. Leo, M. Erkintalo, S. Coen, I. Ricciardi, M. De Rosa, S. Wabnitz, and M. Erkintalo: Singlyresonant second-harmonic generation frequency combs, in preparation, 2016

[10] T. Hansson, F. Leo, M. Erkintalo, J. Anthony, S. Coen, I. Ricciardi, M. De Rosa, and S. Wabnitz: Single envelope equation modelling of multi-octave comb arrays in microresonators with quadratic and cubic nonlinearity, arXiv 1602.08087, 2016.

[11] A. V. Buryak, P. D. Trapani, D. V. Skryabin, and S. Trillo: Optical solitons due to quadratic nonlinearities: From basic physics to futuristic applications, Phys. Rep., vol. 370, 63 (2002). 